\title{
SUPERDENSE MASSIVE GALAXIES IN WINGS LOCAL CLUSTERS
}

\author{
T. Valentinuzzi ${ }^{1}$, J. Fritz 2 , B. M. Poggianti ${ }^{2}$, A. Cava ${ }^{3,4}$, D. Bettoni ${ }^{2}$, G. Fasano ${ }^{2}$, M. D’Onofrio ${ }^{1}$, W. J. Couch ${ }^{5}$,

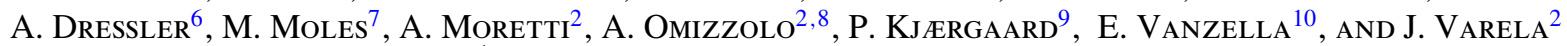 \\ ${ }^{1}$ Astronomical Department, University of Padova, Italy \\ ${ }^{2}$ INAF-Astronomical Observatory of Padova, Italy \\ ${ }^{3}$ Instituto de Astrofisica de Canarias La Laguna, Spain \\ ${ }_{5}^{4}$ Departamento de Astrofisica, Universidad de La Laguna, E-38205 La Laguna, Tenerife, Spain \\ ${ }^{5}$ Center for Astrophysics and Supercomputing, Swinburne University of Technology, Australia \\ ${ }^{6}$ The Observatories of the Carnegie institution of Washington, Pasadena, CA, USA \\ ${ }^{7}$ Instituto de Astrofisica de Andalucia, Granada, Spain \\ ${ }^{8}$ Specola Vaticana, 00120 Vatican City, Holy See \\ ${ }^{9}$ Copenhagen University Observatory, The Niels Bohr Institute for Astronomy, Physics and Geophysics, Denmark \\ ${ }^{10}$ INAF-Astronomical Observatory of Trieste, Italy \\ Received 2009 July 14; accepted 2010 January 14; published 2010 February 26
}

\begin{abstract}
Massive quiescent galaxies at $z>1$ have been found to have small physical sizes, and hence to be superdense. Several mechanisms, including minor mergers, have been proposed for increasing galaxy sizes from high- to low-z. We search for superdense massive galaxies in the WIde-field Nearby Galaxy-cluster Survey (WINGS) of $\mathrm{X}$-ray selected galaxy clusters at $0.04<z<0.07$. We discover a significant population of superdense massive galaxies with masses and sizes comparable to those observed at high redshift. They approximately represent $22 \%$ of all cluster galaxies more massive than $3 \times 10^{10} M_{\odot}$, are mostly S0 galaxies, have a median effective radius $\left\langle R_{e}\right\rangle=1.61 \pm 0.29 \mathrm{kpc}$, a median Sersic index $\langle n\rangle=3.0 \pm 0.6$, and very old stellar populations with a median mass-weighted age of $12.1 \pm 1.3$ Gyr. We calculate a number density of $2.9 \times 10^{-2} \mathrm{Mpc}^{-3}$ for superdense galaxies in local clusters, and a hard lower limit of $1.3 \times 10^{-5} \mathrm{Mpc}^{-3}$ in the whole comoving volume between $z=0.04$ and $z=0.07$. We find a relation between mass, effective radius, and luminosity-weighted age in our cluster galaxies, which can mimic the claimed evolution of the radius with redshift, if not properly taken into account. We compare our data with spectroscopic high- $z$ surveys and find that - when stellar masses are considered - there is consistency with the local WINGS galaxy sizes out to $z \sim 2$, while a discrepancy of a factor of 3 exists with the only spectroscopic $z>2$ study. In contrast, there is strong evidence for a large evolution in radius for the most massive galaxies with $M_{*}>4 \times 10^{11} M_{\odot}$ compared to similarly massive galaxies in WINGS, i.e., the brightest cluster galaxies.
\end{abstract}

Key words: galaxies: clusters: general - galaxies: evolution - galaxies: fundamental parameters - galaxies: structure

Online-only material: color figures

\section{INTRODUCTION}

In the last years high- $z$ studies have uncovered a considerable number of massive galaxies with relatively small effective radii (see, among others, Daddi et al. 2005; Trujillo et al. 2006, 2007; Toft et al. 2007; Zirm et al. 2007; Buitrago et al. 2008; Cimatti et al. 2008; van Dokkum et al. 2008; Saracco et al. 2009; van der Wel et al. 2008), or, in other words, superdense galaxies ${ }^{11}$ (hereafter, SDGs). Although the data sets and methodologies are quite different, they all agree on the fact that a population of massive and compact passive galaxies at $z>1$ does exist, with sizes a factor of at least 3 less that their low- $z$ counterparts of the same mass.

Other studies, using different samples extracted from the Sloan Digital Sky Survey (SDSS), have found a complete absence of such galaxies with old stellar population ages (see, e.g., Shen et al. 2003; Trujillo et al. 2009). This implies the necessity of an evolution in radius with redshift, and it is often considered a proof that these high- $z$ SDGs have undergone significant (minor and/or major) merging events along their histories.

\footnotetext{
11 As far as physical densities are concerned, the galaxies under investigation here are thought not to be extreme (see, e.g., Bezanson et al. 2009; Hopkins et al. 2009a).
}

More recently, Taylor et al. (2009) identify $63 M_{*}>5 \times$ $10^{10} M_{\odot}$ red sequence $z \sim 0.1$ SDG local candidates which are smaller than the median mass-size relation by a factor of at least 2 . These local galaxies have sizes which are compatible with many of the high- $z$ ones, but they seem anyway larger (by a factor of $\sim 2$ ) than the most distant $z \geqslant 2$ and massive $M_{*} \geqslant 10^{11} M_{\odot}$ galaxies recently discovered (see, e.g., Buitrago et al. 2008; van Dokkum et al. 2008; Damjanov et al. 2009).

Different scenarios have been proposed to explain the compactness of these galaxies and their subsequent evolution. One of the simplest ideas is to assume that high- $z$ masses and/or $R_{e}$ measurements, are incorrect. It is true that systematic effects can easily pollute these measurements (see, e.g., van Dokkum et al. 2008; Bezanson et al. 2009), such as low signal-to-noise ratios $(\mathrm{S} / \mathrm{Ns})$, limitations in resolution, uncertainty on the initial mass function (IMF), models and spectral energy distribution (SED) fitting, etc. A recent study of van Dokkum et al. (2009), analyzing a $29 \mathrm{hr}$ exposure spectra of one of their nine high- $z$ SDGs, has found a very high velocity dispersion $\left(\sim 500 \mathrm{~km} \mathrm{~s}^{-1}\right)$, which is consistent with its compact nature and stellar mass from SED fitting. Obviously, this is only one case, and it must be confirmed, but it gives an indication that measurements errors may not be the explanation for the existence of these high- $z$ massive compact objects. 
On the other hand, Mancini et al. (2009), over the 2 deg COSMOS field, select 12 quiescent massive galaxies at $z \sim 1.5$, and found sizes mostly compatible with the local mass-radius relation. Based on mocked images of high- $z$ galaxies initially laying on the local mass-radius relation, they claim that size measures performed on low $\mathrm{S} / \mathrm{N}$ images are likely to give systematically lower $R_{e}$.

By studying a sample of brightest cluster galaxies (BCGs), based on considerations on the evolution of the mass function with redshift and the merging rates in numerical simulations, Bernardi (2009) claims that the main mechanism to let galaxies increase their radius without gaining too much in mass is through minor mergers. This is in general interpreted as an independent indication that very old galaxies have increased their size during their evolution by means of minor merger. However, these findings are not conclusive, as it is not clear whether mergers alone can efficiently puff up galaxies by the required amount, as many parameters are involved in this mechanism (see, among others, Khochfar \& Silk 2006; van der Wel et al. 2009; Joung et al. 2009). Furthermore, this mechanism could be efficient only for BCGs, becoming less and less relevant for other kind of galaxies.

Another viable explanation for puffing up galaxies might be connected with the quasar phase that these galaxies have likely undergone from $z \sim 2.5$ to $z \sim 1$ (Fan et al. 2008), which caused a dramatic mass loss with consequent expansion of the galaxy. However, such expansion would take place at most in a few dynamical times $\left(\sim 8 \times 10^{8} \mathrm{Gyr}\right)$, causing only a few systems to be caught as quiescent and still compact (see Mancini et al. 2009).

Whatever the evolution mechanism, local clusters could be an ideal place for SDGs, as they probably reside in very dense environments at high redshift too; this is supported by the strong clustering $\left(R_{o} \approx 8-10 \mathrm{Mpc}\right)$ of quiescent ${ }^{12}$ compact high- $z$ galaxies (Cimatti et al. 2008, and references therein). At least a fraction of these objects may have survived till recent cosmic epochs; some models predict that $10 \%$ of galaxies have had no significant transformations since $z \sim 2$ (Hopkins et al. 2009b, and references therein). Hence, it is plausible that a certain number of very old compact galaxies are found in local galaxy clusters. In this contest, the WIde-field Nearby Galaxy-clusters Survey (WINGS; Fasano et al. 2006) is a suitable survey where to search for such objects.

The layout of this paper is as follows. In Section 2, we describe the data set we used to search for SDGs. In this section, particular care is given to the homogenization of our data with literature data, and some caveats are discussed. In Section 3, we present our sample of compact galaxies. In Section 4, we discuss the comparison with high- $z$ data and the selection effects that occur when considering old stellar populations at high- $z$, and give number densities and frequencies. In Section 5, we discuss the local SDSS mass-radius relation used by high- $z$ studies as a local reference and some reasons why recent works may have missed local counterparts to massive high- $z$ compact galaxies. In Section 6, we describe in more detail all photometric, spectroscopic, and intrinsic properties of our compact sample, and finally we draw our conclusions in Section 7.

Throughout this paper, we will use the cosmology $\left(H_{0}, \Omega_{m}\right.$, $\left.\Omega_{\lambda}\right)=(70,0.3,0.7)$.

\footnotetext{
12 High- $z$ galaxies are considered quiescent when their luminosity-weighted age is $\geqslant 1.5 \mathrm{Gyr}$; we call quiescent WINGS cluster members with luminosity-weighted ages $\geqslant 10 \mathrm{Gyr}$, i.e., quiescent at $z \sim 1.5$.
}

\section{THE DATA SET}

The galaxies examined in this paper are part of the WINGS (Fasano et al. 2006). WINGS ${ }^{13}$ is a multiwavelength survey especially designed to provide the first robust characterization of the photometric and spectroscopic properties of galaxies in nearby clusters, and to determine the variations of these properties as a function of galaxy mass and environment.

Clusters were selected in the X-ray from the ROSAT Brightest Cluster Sample and its extension (Ebeling et al. 1998, 2000) and the X-ray Brightest Abell-type Cluster sample (Ebeling et al. 1996). WINGS clusters cover a wide range of velocity dispersion $\sigma_{\text {clus }}$, typically between 500 and $1100 \mathrm{~km} \mathrm{~s}^{-1}$, and X-ray luminosity $L_{X}$, typically $(0.2-5) \times 10^{44} \mathrm{erg} \mathrm{s}^{-1}$.

The survey core, based on optical $B, V$ imaging of 78 nearby $(0.04<z<0.07)$ galaxy clusters (Varela et al. 2009), has been complemented by several ancillary projects: (1) a spectroscopic follow-up of a subsample of 48 clusters, obtained with the spectrographs WYFFOS@WHT and 2dF@AAT (Cava et al. 2009); (2) near-infrared $(J, K)$ imaging of a subsample of 28 clusters obtained with WFCAM@UKIRT (Valentinuzzi et al. 2009); (3) $U$ broad- and $\mathrm{H}_{\alpha}$ narrowband imaging of subsamples of WINGS clusters, obtained with wide-field cameras at different telescopes (INT, LBT, Bok; see A. Omizzolo et al. 2010, in preparation).

In the following, we will use only spectroscopically confirmed members of the subset of WINGS clusters that have an average spectroscopic completeness larger than 50\% (21 out of 78 clusters). Our completeness is essentially independent of distance to the cluster center for most clusters, and is completely independent of galaxy radius. The only criterion used for spectroscopic selection was galaxy magnitude (Cava et al. 2009), but given that separate configurations were used to take spectra of bright and faint galaxies and also due to fiber collision effects, completeness turns out to be rather flat even with magnitude for most clusters.

In this paper, WINGS results are compared with literature data at $0.9<z<2.5$. Several studies have investigated the sizes of distant quiescent galaxies, but we only consider here high- $z$ data sets based on spectroscopic redshifts that give high-quality masses and sizes, while other works that used photometric redshifts (i.e., Toft et al. 2007; Zirm et al. 2007; Buitrago et al. 2008) are not included in the present study. We use the following data sets: HUDF (Daddi et al. 2005), MUNICS (Trujillo et al. 2006), MUSYC (van Dokkum et al. 2008), Saracco et al. (2009), GMASS (Cimatti et al. 2008), van der Wel et al. (2008), and Damjanov et al. (2009). The data, methods of analysis and, most importantly, selection criteria for these samples clearly differ from one study to another. In the comparison amongst different samples, it is of paramount importance to account for differences in models and IMF adopted (see following sections). We stress that all of these works, with the exception of van der Wel et al. (2008) that have used a visual early-type morphological classification, have selected their galaxies to have already old (typically 1.5-2 Gyr) stellar populations at that redshift based on SED spectral fitting, line index age dating, absence of significant emission lines, or other spectro-photometric analysis methods.

\subsection{Surface Photometry and Morphology in WINGS}

WINGS effective radii, axial ratios, and Sersic indexes are measured on the $V$-band images with GASPHOT (Pignatelli et al. 2006; M. D'Onofrio et al. 2010, in preparation), an

\footnotetext{
13 Refer to the WINGS Web site for updated details on the survey and its products, http://web.oapd.inaf.it/wings
} 
automated tool which performs a simultaneous fit of the major and minor axis light growth curves with a two-dimensional flattened Sersic law, convolved by the appropriate, spacevarying point-spread function (PSF). In this way, GASPHOT exploits the robustness of the one-dimensional fitting technique, keeping at the same time the capability (typical of the twodimensional approach) of dealing with PSF convolution in the innermost regions.

GASPHOT has proved to be very robust in recovering the best-fitting parameters, and to give the appropriate weight to the external parts of the galaxies, where PSF effects are negligible. Indeed we tested GASPHOT on more than 15,000 simulated and real galaxies, obtaining robust upper limits for the errors of the global parameters of galaxies, even for non-Sersic profiles and blended objects (Pignatelli et al. 2006).

GASPHOT was also tested against the widely used tools GALFIT (Peng et al. 2002) and GIM2D (Marleau \& Simard 1998): it has been found (see, Pignatelli et al. 2006, Section 6) that the performances of these tools are quite similar for large and regular simulated galaxies, while GASPHOT has proved to be more robust for real galaxies with some kind of irregularity or blending, which is a crucial feature when dealing with blind surface photometry of huge galaxy samples. In Section 3.1, we show a comparison of GASPHOT estimates with literature data.

The GASPHOT output effective radius $R_{e}$ value is calculated along the major axis, and for the purposes of this paper is circularized with the usual formula:

$$
R_{e}^{(\mathrm{circ})}=R_{e}^{(\text {major })} \cdot \sqrt{b / a},
$$

where $a$ and $b$ are the major and minor axes of the best-fit model, respectively.

WINGS morphologies are derived from $V$ images using the purposely devised tool MORPHOT (G. Fasano et al. 2010, in preparation). Our approach is a generalization of the nonparametric method proposed by Conselice et al. (2000, see also Conselice 2003). In particular, we have extended the classical CAS (Concentration/Asymmetry/clumpinesS) parameter set by introducing a number of additional, suitably devised morphological indicators, using a final set of 10 parameters. A control sample of 1000 visually classified galaxies has been used to calibrate the whole set of morphological indicators, with the aim of identifying the best subset among them, as well as of analyzing how they depend on galaxy size, flattening and $\mathrm{S} / \mathrm{N}$. The morphological indicators have been combined with two independent methods, a maximum likelihood analysis and a neural network trained on the control sample of visually classified galaxies. The final, automatic morphological classification combines the results of both methods. We have verified that our automatic morphological classification reproduces quite well the visual classification by two of us (A.D. and G.F.). In particular, the robustness and reliability of the MORPHOT results turn out to be comparable with the typical values obtained comparing each other the visual classifications obtained by different (experienced) human classifiers (G. Fasano et al. 2010, in preparation). Although MORPHOT provides a fine classification following the "Revised Hubble Type" de Vaucouleurs (1974), we will use in the following just three broad morphological classes, ellipticals, S0s (together early type) and late type, where the late-type class includes any galaxy later than an S0.

\subsection{Stellar Masses, Ages, and Metallicity}

Stellar masses of WINGS galaxies have been determined by fitting the optical spectrum (in the range $\sim 3600$ to $\sim 7000 \AA$ ), with the spectro-photometric model fully described in Fritz et al. (2007). All the main spectro-photometric features (such as the continuum flux and shape, and the equivalent widths of emission and absorption lines) are reproduced by summing the theoretical spectra of simple stellar population (SSP) of 13 different ages (from $3 \times 10^{6}$ to $\sim 14 \times 10^{9}$ years).

Dust extinction is allowed to vary as a function of SSP age, in a screen uniformly distributed in front of the stars. The Galactic extinction law follows Cardelli et al. (1989) scheme, with $R_{V}=3.1$. As explained in detail in Fritz et al. (2007), for the fit we use a fixed metallicity, exploring three values: $Z=0.004, Z=0.02$, and $Z=0.05$. The adopted star formation histories and stellar masses refer to the model with the metallicity value that provides the lowest $\chi^{2}$. The lowest $\chi^{2}$ for the great majority of our SDGs yields either solar or supersolar metallicities.

SSP spectra are built using Padova evolutionary tracks and the observed MILES spectral library (Sanchez-Blazquez 2004; Sánchez-Blázquez et al. 2006) for ages older than $10^{9}$ years, complemented by the Jacoby et al. (1984) library for young SSPs, and in the UV and infrared by means of the Kurucz theoretical library. Nebular emission is also included, modeled with values that are typical of $\mathrm{H}$ II regions: this significantly affects spectra of SSPs younger than $\sim 2 \times 10^{7}$ years.

\subsubsection{Stellar Masses}

There are three main definitions of galaxy stellar mass derived by means of spectral synthesis (see, e.g., Longhetti \& Saracco 2009, for details):

1. the initial mass of the SSP, i.e. the mass of all the SSP stars at the moment of their formation. This mass does not depend on the SSP's age, being fixed once and for all;

2. the mass locked into stars, including stellar remnants, at any time;

3. the mass of stars that are still in the nuclear burning phase (i.e., no remnants included), at any time.

The difference between these definitions (up to a factor of 2 from definition 1 to definition 3, depending on several model parameters, such as, e.g., the IMF) is a function of the SSP age, as the fraction of gas which is returned to the interstellar medium and the fraction of stars that evolve into remnants increase with time. In this paper, we only use masses derived from definition 2.

Our spectra are taken within a 2 arcsec aperture fiber. For the purpose of computing total stellar masses and star formation rates, model spectra are rescaled to match the observed total $V$ magnitude, i.e., the SExtractor MAG_AUTO from Varela et al. (2009). This assumes that color gradients within galaxies are negligible. In order to take into account color gradient effects, we apply a correction of $\Delta(B-V)$ dex $^{14}$ to our masses. This correction is based on the prescription given in Bell \& de Jong (2001).

\subsubsection{Mass- and Luminosity-weighted Ages}

From our spectral analysis, it is possible to derive an estimate of the average age of the stars in a galaxy. Following the definition of Cid Fernandes et al. (2005), we compute the luminosity-weighted age (Lw-age) by weighting the age of

\footnotetext{
${ }^{14} \Delta(B-V)=(B-V)_{\text {fiber }}-(B-V)_{10 \mathrm{kpc}}$, where $10 \mathrm{kpc}$ is the physical aperture diameter. The median correction in mass for galaxies with $M_{*}>3 \times 10^{10} M_{\odot}$ is $-0.05 \mathrm{dex}$
} 


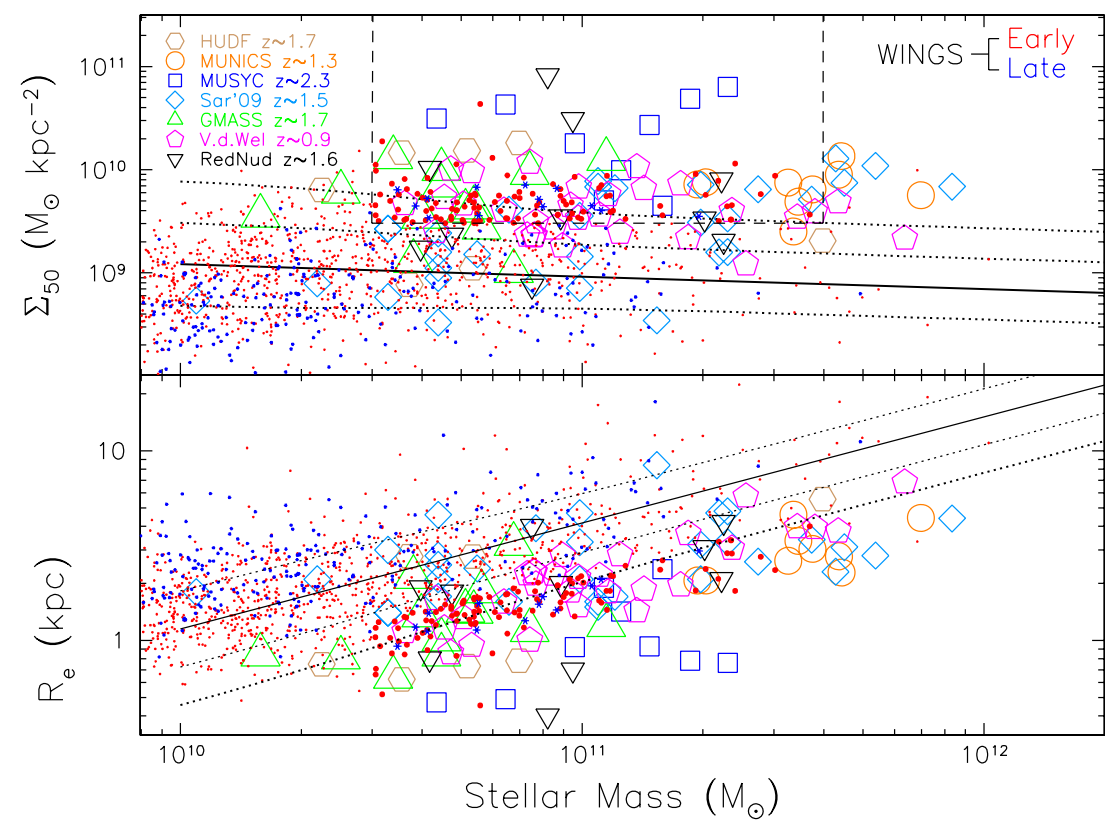

Figure 1. Circularized effective radius $R_{e}$ and the mass density inside $R_{e}$ as a function of stellar mass for all WINGS galaxies with $M_{*} \geqslant 10^{10} M_{\odot}$, for the subsample of 21 clusters considered (see the text). Blue and red tiny dots are late- (later than S0) and early-type (ellipticals and S0s) WINGS cluster galaxies, respectively. The region corresponding to our SDGs definition is delimited by the dashed lines in the top panel. The corresponding larger blue stars and red dots mark the WINGS SDGs. The black solid line is the SDSS-DR4 (Shen et al. 2003) relation with dotted $1 \sigma$ and $2 \sigma$ lines. Open symbols are SDGs from high- $z$ studies, see the text for references. (A color version of this figure is available in the online journal.)

each SSP composing the integrated spectrum with its bolometric flux. This provides an estimate of the average age of the stars weighted by the light we actually observe. A mass-weighted age (Mw-age) is computed in a similar way: each SSP age is weighted with its mass value. The mass-weighted age is the "true" average age of the galaxy's stars. For our sample of cluster galaxies mass-weighted ages are systematically larger ( $\sim 2$ Gyr) than luminosity-weighted ages.

\subsubsection{IMF and Model Differences}

The spectro-photometric analysis performed on the WINGS spectra was done assuming a Salpeter (1955) IMF with masses in the range $0.15-120 M_{\odot}$. We then rescale both our values and all those from the literature to a Kroupa (2001) IMF, with masses in the range $0.01-50 M_{\odot}$. It is also extremely important to properly match models that use different treatments of the thermally pulsating asymptotic giant branch phase (TP-AGB). When needed, i.e., for high- $z$ literature galaxies, all the mass values were rescaled in order to match those obtained with the Maraston (2005) models, applying a correction of 0.15 dex to the masses derived from Bruzual and Charlot models, as prescribed by Cimatti et al. (2008). This difference in mass is strongly depending on the stellar population age, becoming practically negligible for ages older than $2 / 3 \mathrm{Gyr}$, i.e., it is irrelevant for local cluster galaxies, which are extremely old.

\section{RESULTS: WINGS SUPERDENSE GALAXIES}

In the bottom panel of Figure 1, we plot the circularized effective radius $R_{e}$ as a function of stellar mass for spectroscopically confirmed WINGS cluster members with stellar masses $M_{*} \geqslant 10^{10} M_{\odot}$. In the upper panel, we plot the mean mass surface density inside $R_{e}$

$$
\Sigma_{50}=\frac{0.5 M_{*}}{\pi R_{e}^{2}}
$$

as usually defined by other authors (see, among others, Cimatti et al. 2008; van der Wel et al. 2008).

After rescaling all the masses to the same Kroupa (2001) IMF and models (see previous section), we overplot high- $z$ data from the literature as large open symbols. It can be seen that literature data cover a large range of masses and radii/densities, and a considerable fraction does not even reside in the highest mass-density locus.

We choose the SDGs WINGS subsample (larger blue stars and red dots) in order to match as much as possible the position of high- $z$ SDG data in Figure 1 (region inside the dashed lines), applying the following criteria:

$$
\begin{gathered}
3 \times 10^{10} M_{\odot} \leqslant M_{*} \leqslant 4 \times 10^{11} M_{\odot}, \\
\Sigma_{50} \geqslant 3 \times 10^{9} M_{\odot} \mathrm{kpc}^{-2} .
\end{gathered}
$$

We exclude from the SDG sample the BCGs and other galaxies more massive than $4 \times 10^{11} M_{\odot}$, as they may have a more complex formation history (dry/wet minor/major merger) which could, in principle, pollute our analysis (see Section 5 and Figure 5).

The resulting sample consists of 134 galaxies. Analyzing individually both images and spectra of this sample, we decided to exclude 12 of them because of close companions, bad chip regions, or low $\mathrm{S} / \mathrm{N}$ spectra. From here on we refer to the remaining 122 objects as the WINGS SDGs sample that includes nearly $22 \%$ of all cluster members with $M_{*} \geqslant 3 \times 10^{10} M_{\odot}$; we recall here that we are using only the spectroscopic confirmed members of a subset of 21 (all from the southern hemisphere) out of 78 WINGS clusters. Regarding their morphologies, 31 of them are ellipticals, $78 \mathrm{~S} 0$ s, and 13 late-type galaxies.

In Figure 1, we also draw the Shen et al. (2003) SDSS low- $z$ relation for early-type galaxies, selected to have a Sersic index $n>2.5$ (full black line, with $1 \sigma$ and $2 \sigma$ as dotted lines), 

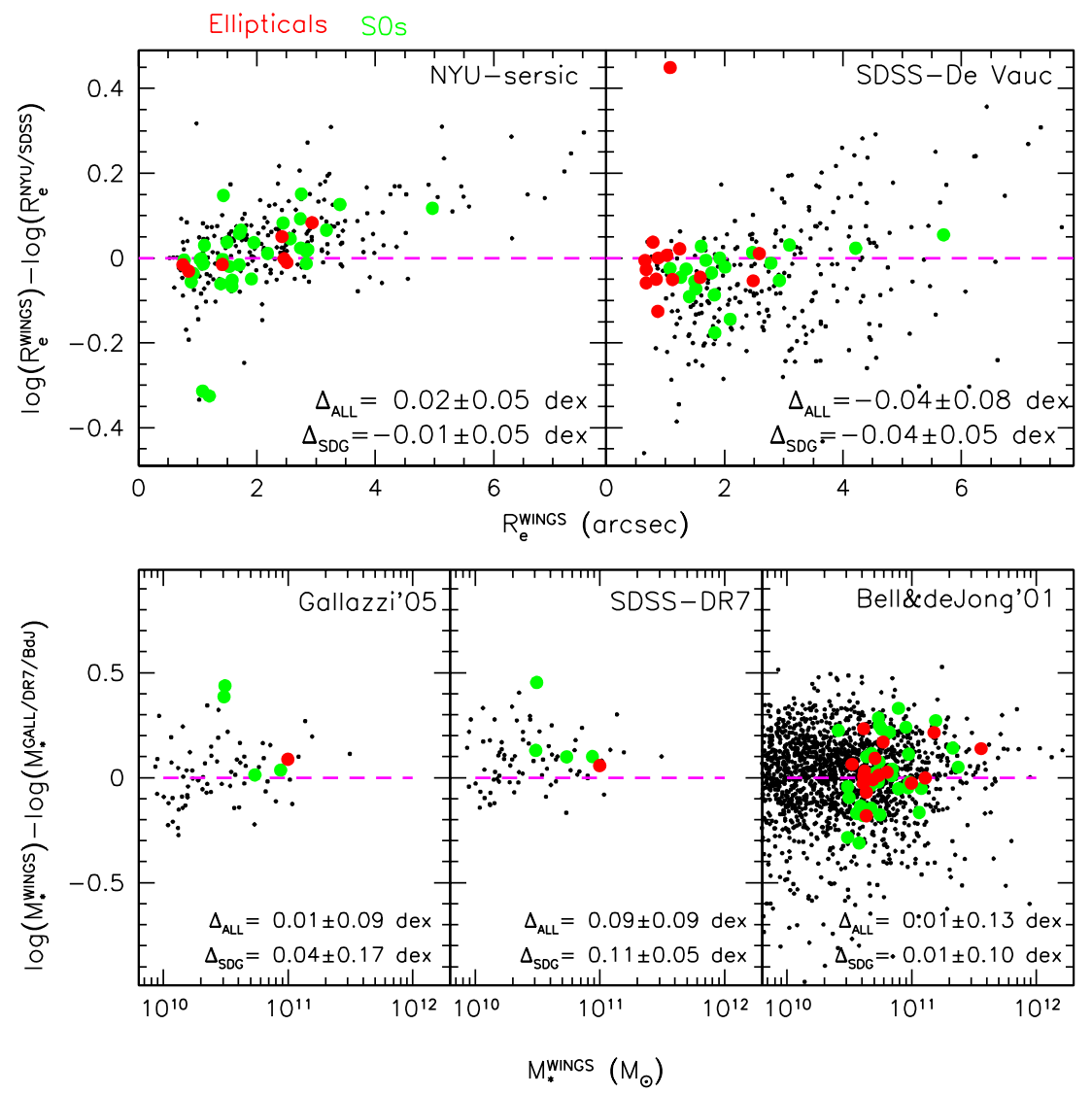

Figure 2. Consistency check for WINGS sizes and masses. Only early-type WINGS spectroscopically confirmed member galaxies are shown. Big colored dots are SDGs, red for ellipticals and green for S0's. Top panels: difference in $R_{e}$ estimates (dex) between GASPHOT, NYU-VAGC (Sersic fit, values from spectroscopic DR7 catalogs), and SDSS-DR7 (de Vaucoleurs fit, from photometric DR7 catalogs) values in arcseconds (see the text for details). Bottom panels: difference in mass (dex) between WINGS estimates and Gallazzi et al. (2005), SDSS-DR7 and Bell \& de Jong (2001).

(A color version of this figure is available in the online journal.)

commonly used by high- $z$ studies as a reference point for the local mass-radius relation. We note at this point that the WINGS SDGs sample is found at more than $2 \sigma$ from the mean SDSS sample. In Section 5, we will discuss the local SDSS relation in more detail.

It is clear that in the WINGS data set we do find a considerable number of galaxies with masses, radii, and mass densities typical of high- $z$ SDGs. The only high- $z$ samples that stand out for their extreme densities and low radii are six of the nine galaxies from van Dokkum et al. (2008) and two of the 10 galaxies from Damjanov et al. (2009) that do not have local WINGS counterparts. We will discuss further these cases in the following section.

We note that for masses $M_{*} \leqslant 3 \times 10^{10} M_{\odot}$ there is a significant decrease in the frequency of SDGs (Figures 1 and 3): we checked whether this is due to completeness effects that could result in systematically missing effective radius and/or morphology measurements of small objects for low masses/luminosities, but this is not the case. We speculate that this rapid decrease in number is an indication that a minimum threshold in mass is required to form compact galaxies.

\subsection{Comparison of Sizes and Masses with Literature Data}

It is widely known that stellar masses based on spectrophotometric models have typical errors of $\sim 0.2$ dex. A crucial issue is to ensure that low- $z$ masses are comparable to high$z$ ones. Dynamical masses from integral field spectroscopy or virial masses from central velocity dispersions can, in principle, be the solution for this kind of studies (see, e.g., van der Wel et al. 2008), but it is very time consuming and very difficult to apply at $z>2$.

We calculated the virial masses of our WINGS galaxies from the velocity dispersions we found from literature: they are $\sim 0.14$ dex heavier than our Kroupa (2001) IMF stellar masses (the same offset was recovered by Cappellari et al. 2006). Some $20 \%$ of our galaxies (either compact or normal ones) present an excess of stellar mass. This can be explained by the large uncertainties involved and by the need of accurate dynamical models, as thoroughly explained in Cappellari et al. (2006). Integral field spectroscopy of our SDGs will further clarify this issue.

The SDGs central velocity dispersions $\sigma_{o}$ range from 100 up to $300 \mathrm{~km} \mathrm{~s}^{-1}\left(\left\langle\sigma_{o}\right\rangle=180 \pm 30 \mathrm{~km} \mathrm{~s}^{-1}\right)$. These values are significantly smaller than the value of $\sim 500 \mathrm{~km} \mathrm{~s}^{-1}$ presented by van Dokkum et al. (2009) for a compact galaxy at $z=2.2$ drawn from their MUSYC sample. Instead, they are surprisingly in agreement with measurements at $z>1.5$ by Cenarro \& Trujillo (2009), recently confirmed by Cappellari et al. (2009) on GMASS galaxies in the redshift range $1.4 \leqslant z \leqslant 2.0$.

In the top panel of Figure 2, we show the comparison between WINGS early-type effective radii estimates and both NYU-VAGC ${ }^{15}$ (Sersic fit, left panel, based on the spectroscopic SDSS-DR7 catalogs) and SDSS ${ }^{16}$ (de Vaucoleurs fit, right

\footnotetext{
15 http://sdss.physics.nyu.edu/vagc/

$16 \mathrm{http}: / /$ cas.sdss.org/dr7/en/tools/crossid/crossid.asp
} 
panel, based on the photometric SDSS-DR7 catalogs) values. We plot only early-type WINGS spectroscopic members with $M_{*}>3 \times 10^{10} M_{\odot}$, and for this comparison we use all WINGS survey data (not only the 21 clusters of this paper). Big colored dots are the WINGS SDGs in common, red for ellipticals and green for S0's. It is apparent that most of the points lie in the region within 0.1 dex difference, and the medians do not show important offsets. There are some $(\sim 5 \%)$ that seem to show an underestimation of GASPHOT radii with respect to NYU-VAGC and SDSS. We checked "by eye" all these cases and found that many of these present a disturbing star in proximity of the galaxy, have a close galaxy pairs or groups, or a galaxy size below (at the limit of) the SDSS resolution. Due to the robustness of GASPHOT in these peculiar conditions, and to the deeper and higher-resolution images of WINGS (for details, see Fasano et al. 2006), we are tempted to think that our estimates are more reliable even in those cases. More interestingly, SDGs galaxies have compatible $R_{e}$ measurements, as only one case shows a WINGS radius much lower than the literature one. Again, this is an S0 strongly contaminated by a secondary object.

In the bottom panels of Figure 2, we show that our masses are in good agreement with the SDSS-DR4 estimates from Gallazzi et al. (2005), even though the scatter is high $(\sim 0.15 \mathrm{dex})$, while we find an offset of $\sim 0.09$ dex with SDSS-DR7 masses. ${ }^{17}$ Here, we consider only the 21 clusters used in this paper (all from the southern hemisphere) that have both high-quality photometry and reliable masses based on high-quality spectroscopic data. Only three of the 21 clusters are in common with SDSS (namely, A2399, and, partly, A119 and A957x which are covered at the $40 \%$ level), therefore the number of SDG galaxies that can be used for this comparison is small. Our mass estimates agree with masses calculated with $(B-V)$ color and total $V$-band fluxes with the recipe of Bell \& de Jong (2001). We note here that Shen et al. (2003) use SDSS-DR4 Kauffmann et al. (2003) masses which are lower by a factor of $0.07 \mathrm{dex}$ and of 0.13 dex when compared to Gallazzi et al. (2005) and our masses, respectively.

As a further check we have run the popular Hyperzmass software (see Bolzonella et al. 2000) on $B, V$ filters, finding no appreciable offset with WINGS masses.

More interestingly, these comparisons and tests show that the SDGs are not systematically extremes, giving more reliability to our conclusions on compactness of massive local WINGS cluster galaxies.

\section{COMPARISON WITH HIGH- $z$ : THE IMPORTANCE OF STELLAR AGE-SELECTION EFFECTS}

Though, as shown in the previous section, there are low- $z$ superdense counterparts to the high- $z$ SDGs, and they represent a significant fraction of the local cluster massive galaxy population, it remains to be addressed whether the prevalence of SDGs among the observed high- $z$ galaxies requires a size evolution in a significant fraction of all massive galaxies.

In Figure 3, we show the combined effect of stellar mass and effective radius in determining the stellar age of a galaxy. The grayscale corresponds to luminosity-weighted ages (see legend for details): at fixed mass, smaller galaxies are older, while for a fixed radius, more massive galaxies are older. The same general trend is preserved if mass-weighted ages are used, so that even the formation epoch of the bulk of the stellar mass of these objects depends simultaneously on stellar mass and radius.

\footnotetext{
17 http://www.mpa-garching.mpg.de/SDSS/DR7/Data/stellarmass.html
}

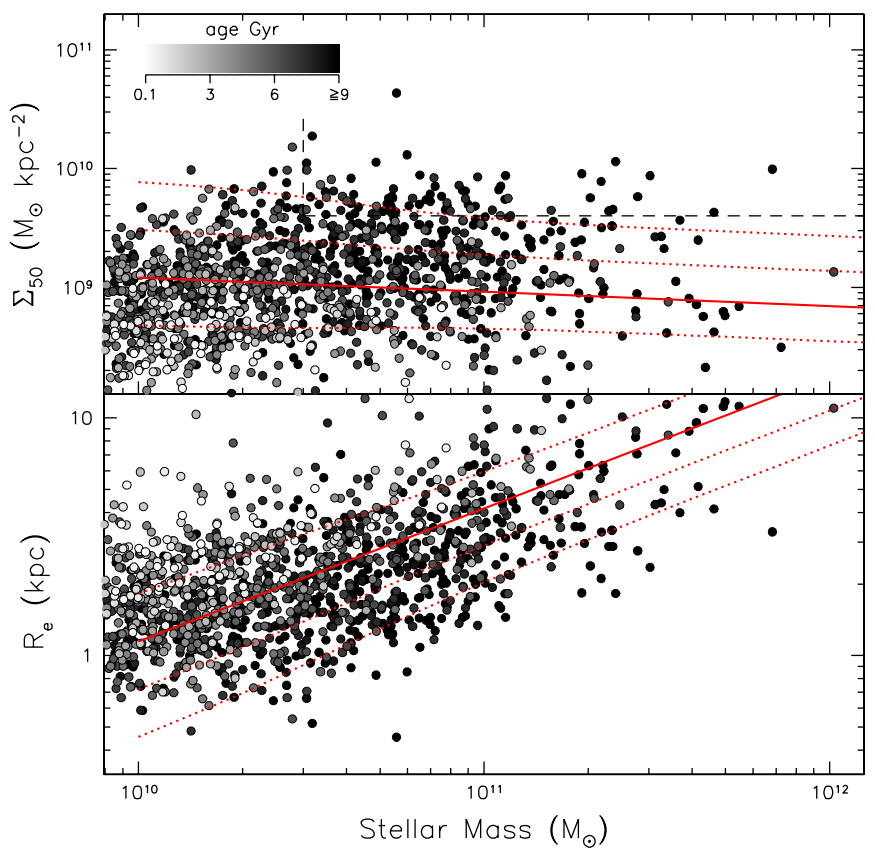

Figure 3. Same as Figure 1 but only for WINGS galaxies and with grayscale filled dots showing luminosity-weighted ages. The black color is assigned to luminosity-weighted age $\geqslant 9 \mathrm{Gyr}$ (equivalent to being quiescent at $z \sim 1.3$ ). More massive galaxies tend to have older ages and, at a given mass, galaxies with smaller radii are older (see Section 4).

(A color version of this figure is available in the online journal.)

Comparing the sizes of massive high- $z$ galaxies with the SDSS Shen et al. (2003) relation, several authors have claimed the necessity of an evolution of the size of such galaxies with redshift, at least of a factor of $3(0.5 \mathrm{dex})$. While we will address the necessity to "properly" calibrate the local massradius relation in the next section, we want now to focus on the effect of a luminosity-weighted age selection. All high- $z$ studies shown in Figure 1 have selected their galaxies to be "old" on the basis of their stellar population properties (either SED fitting, lack of significant emission lines, spectral features, etc.), which translates into selecting galaxies with a luminosity-weighted age at least 1.5-2 Gyr old at the redshift they are observed.

In Figure 4, we show the median $R_{e}$ of WINGS galaxies (filled dots) with luminosity-weighted ages older (by $\geqslant 1.5 \mathrm{Gyr}$ ) than the age corresponding to the plotted redshift. The three panels refer to three stellar mass intervals, chosen to match the various high- $z$ samples and to have a sufficient number of galaxies for statistics. The mean sizes of high- $z$ literature data are plotted in color symbols (see the legend). The dotted points have less than three galaxies, while all other have at least three galaxies in each interval. The magenta pentagons are the van der Wel et al. (2008) data, who use virial masses on the basis of central velocity dispersion measurements; we correct these masses with a mean 0.15 dex contribution of dark matter in order to be globally compatible with our stellar masses. Even with this correction, the van der Wel et al. (2008) "stellar-like" masses we derive may not be completely consistent with the stellar mass estimates of all other samples used in this paper, so the comparison with van der Wel et al. (2008) should be treated with caution.

On top of the main well-known correlation of radius with mass (more massive galaxies are on average bigger in size), we find a noticeable decrease of the median radius with increasing redshift when galaxies are selected to be old at that redshift. The 


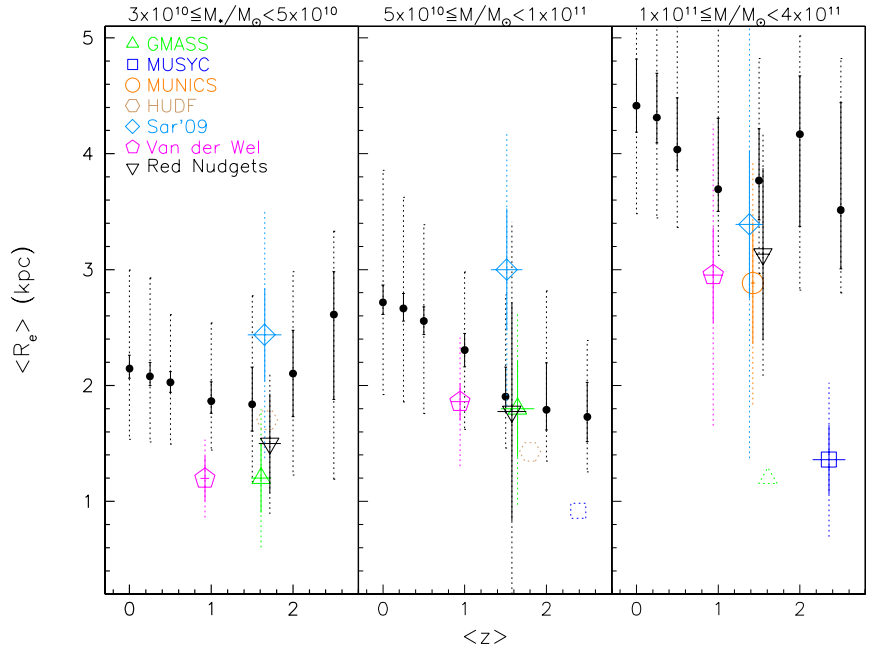

Figure 4. Median $R_{e}$ of WINGS galaxies (filled dots) that stopped forming stars (i.e., with luminosity-weighted ages older by) at least $>1.5 \mathrm{Gyr}$ before the redshift plotted. Three stellar mass intervals are considered. High- $z$ literature data are plotted as color symbols (see legend). WINGS error bars represent the errors on the median size, while the dotted error bars are the upper and the lower quartiles of the corresponding distribution. Due to the scarcity of high- $z$ data, the high- $z$ data points are mean values with their corresponding rms scatter (dotted error bars) and rms of the mean (full error bars). When the mean is done on less than three galaxies, the symbol is dotted, to show its low statistical significance.

(A color version of this figure is available in the online journal.)

older the stellar population is selected, the smaller the median effective radius.

Stellar age-selection effects are therefore important: high- $z$ studies find preferably compact galaxies because they select them to be old (i.e., to have only old stars). Assuming the sizes of today cluster massive galaxies to be representative of the sizes of all massive galaxies regardless of environment, we speculate that if high- $z$ studies would include galaxies of all luminosityweighted ages (young and old), they would find median effective radius values compatible with the global WINGS mass-radius relation, at least as far as $z \sim 2$ (i.e., our limit of resolution in age).

In other words, a galaxy which is seen to be star forming (and with larger sizes, see, e.g., Kriek et al. 2009) at, say, $z \sim 2$ will obviously not be included in the high- $z$ passive galaxy samples, which will have a smaller size. As we reach $z \sim 0$, those larger star-forming high- $z$ galaxies that have become passive in the meantime, cause the local median size of passive galaxies to be apparently larger than that at high- $z$. The correct thing to do is to compare those high- $z$ sizes with the sizes of local galaxies which were quiescent at those high redshifts.

Figure 4 shows that, in general, no evolution in radius is required for most of the high- $z$ samples we consider in the present study. The majority of high- $z$ data points are consistent within $1 \sigma$ with the WINGS estimates, even when the smallest error bars (errors on the means) are considered.

The van der Wel et al. (2008) data points, which we recall are obtained from dynamical masses, and therefore on stellar mass estimates that are not homogeneous with all other samples, show at most a factor 1.2-1.5 of evolution in size, much lower than the factor 3 claimed in the literature.

We note that when Saracco et al. (2009) divide their high- $z$ sample in two classes of galaxies characterized by old (Lw-age $\sim 3.5 \mathrm{Gyr}$ ) and young (Lw-age $\sim 1.5 \mathrm{Gyr}$ ) stellar populations, they find younger galaxies to have sizes compatible with the local mass-radius relation, in contrast with older galaxies that have smaller radii. The dependence of galaxy stellar age on galaxy size, at a fixed mass, must clearly be already established at $z \sim 1.5$. Our results confirm recent findings of other authors: Shankar \& Bernardi (2009) have recently pointed out that, at fixed mass, smaller galaxies have older stellar populations, and the same conclusion is implicit in Graves et al. (2009).

The present study demonstrates that comparing the sizes of passive galaxy samples at high- $z$ with local samples, can mimic a fictitious evolution of radius with redshift, if the effects of the stellar age selection are not properly taken into account. In contrast, our results point toward an overall consistency between the sizes of high- $z$ quiescent and low- $z$ old massive galaxies, with at most a very mild evolution in size, as far as cluster galaxies are concerned. Considering single points in Figure 4 instead of the global tendency, the maximum amount of evolution in size from the present study is a factor of 1.5 , much lower than the claimed factor 3 . In a picture where systematic errors affect high- $z$ measures of sizes (see Mancini et al. 2009) such a factor would be probably easily accommodated, but this will have to be further investigated.

We tested the robustness of our conclusions by using masses with BC03 models instead of MA05, and luminosity-weighted ages calculated for a fixed metallicity (either solar or supersolar) for all galaxies. In all these cases, the conclusions drawn from Figure 4 remain the same.

Our conclusion is challenged by the lowest median sizes of van Dokkum et al. (2008). Such extremely low values of $R_{e}$ are visibly different from all other high- $z$ data. They have median radii 2.5-3 times lower than the global WINGS mass-radius relation. It has to be noted that van Dokkum et al. (2008) extreme cases lay in a section of the plot where we are loosing our model resolution ${ }^{18}$ in age. On the other hand, for such extreme cases systematic effects caused by large distance could be important; for example van Dokkum et al. (2008) discuss some caveats on size estimates of their high- $z$ sample which could give a factor of 2 greater sizes, much more compatible with Figure 4 (see also Mancini et al. 2009). However, it is true that, if more galaxies of such compact nature would be found in the future, they would be candidates of a "growing-radius" class of galaxies which would not be explained by an age-selection effect.

\subsection{Frequency and Number Density}

We now turn to analyze the frequency and number density of WINGS SDGs. We have seen that SDGs represent a sizable fraction $(22 \%)$ of all cluster spectroscopically confirmed members more massive than $3 \times 10^{10} M_{\odot}$. This fraction does not vary using higher-mass cutoff limits, i.e., 5 or $8 \times 10^{10} M_{\odot}$.

We determine the expected total number of SDGs in all WINGS clusters by multiplying the average SDG number per cluster among the 21 clusters considered in this study, corrected for spectroscopic completeness ( $\sim 10 \mathrm{SDG} /$ cluster $)$, by the total number of clusters in the WINGS survey (78).

We then calculate the whole comoving volume associated with the redshift range of WINGS clusters:

$$
V_{\mathrm{WINGS}}=\frac{4 \pi}{3}\left(R_{2}^{3}-R_{1}^{3}\right)(1-\sin b)=5.73 \times 10^{7} \mathrm{Mpc}^{3},
$$

where $b=20^{\circ}$ is the limit in Galactic latitude imposed by the survey to avoid the Galactic disk regions, and $R_{1}=169.8 \mathrm{Mpc}$ and $R_{2}=295.0 \mathrm{Mpc}$ are the distances in our cosmology

\footnotetext{
18 It is very difficult to properly assign an age to galaxies older than 9 Gyr, as
} their spectra are practically the same. 
Table 1

Number Densities of SDGs and Quiescent Galaxies

\begin{tabular}{lcc}
\hline \hline \multicolumn{1}{c}{ Criteria } & $\begin{array}{c}\text { WINGS } \\
\left(10^{-5} \mathrm{Mpc}^{-3}\right)\end{array}$ & $\begin{array}{c}\text { Literature } \\
\left(10^{-5} \mathrm{Mpc}^{-3}\right)\end{array}$ \\
\hline SDGs & $1.31 \pm 0.09$ & $\ldots$ \\
SDGs $M_{*} \geqslant 8 \times 10^{10} M_{\odot}$ & $0.46 \pm 0.05$ & $\ldots$ \\
SDGs LW-age $\geqslant 10 \mathrm{Gyr}(z=1.5)$ & $0.57 \pm 0.06$ & $\ldots$ \\
SDGs $R_{e} \leqslant 1.5 \mathrm{kpc}$ & $0.68 \pm 0.07$ & $\ldots$ \\
Quiescent, $3 \times 10^{10} M_{\odot} \leqslant M_{*} \leqslant 4 \times 10^{11} M_{\odot}$ & $1.55 \pm 0.06$ & $\ldots$ \\
Quiescent $z \sim 2.5, M_{*} \geqslant 10^{11} M_{\odot}$ & $0.50 \pm 0.06$ & Bez $=3.6$ \\
Quiescent $z \sim 1.5,10^{10} \leqslant M_{*} \leqslant 10^{11} M_{\odot}$ & $1.66 \pm 0.10$ & Cimatti $=10$ \\
Quiescent $z>1.5, M_{*} \geqslant 4 \times 10^{10} M_{\odot}$ & $1.80 \pm 0.11$ & Wuyts $=11$ \\
Quiescent $z>1.5, M_{*} \geqslant 10^{11} M_{\odot}$ & $1.09 \pm 0.08$ & Wuyts $=4.5$ \\
\hline
\end{tabular}

Notes. Literature data-Bez: Bezanson et al. 2009; Cimatti: Cimatti et al. 2008; Wuyts: Wuyts et al. 2009. Errors are derived prom Poissonian statistics.

corresponding to the minimum $(z=0.04)$ and maximum $(z=0.07)$ redshifts of our clusters, respectively.

Assuming no SDG is present outside of WINGS clusters in this volume, a very hard lower limit to the SDG number density in the local universe is then $N=1.31 \times 10^{-5} \mathrm{Mpc}^{-3}$ for $M_{*} \geqslant 3 \times 10^{10} M_{\odot}$, and $N=0.46 \times 10^{-5} \mathrm{Mpc}^{-3}$ for $M_{*} \geqslant 8 \times 10^{10} M_{\odot}$ (see Table 1 ).

Considering only the volume effectively probed by the WINGS clusters (a total area of about $25 \mathrm{deg}^{2}$ and an average redshift range $z \pm 0.007$ around each cluster redshift), the SDG number density in clusters turns out to be very high, $N=2.9 \times 10^{-2} \mathrm{Mpc}^{-3}$.

Ideally, we would like to compare the SDG number density we derive with the number density of high- $z$ SDGs, to investigate what fraction of the distant SDGs can have superdense local descendants, consistent with having maintained its size and mass unaltered since $z>1$. Unfortunately, the SDG number density at high- $z$ is not available. The information that several authors provide is the number density of high- $z$ quiescent galaxies (Cimatti et al. 2008; Bezanson et al. 2009; Wuyts et al. 2009), but, as it can be also seen in Figure 1, a large fraction of high- $z$ quiescent galaxies are not superdense.

The number density of high- $z$ quiescent galaxies can be compared with WINGS estimates for galaxies that according to our luminosity-weighted ages should be quiescent (= with stellar ages older than $1.5 \mathrm{Gyr}$ ) at each redshift (Table 1).

Interestingly, nearly $20 \%$ of all high- $z$ quiescent galaxies are found in low- $z$ WINGS clusters, of which about one third is superdense. Several uncertainties and systematics affect this fraction: for example, just by taking all galaxies older that 9 Gyr instead of $10 \mathrm{Gyr}$, the number density of quiescent galaxies in clusters increases by $50 \%$. Moreover, we stress that the number of both SDGs and quiescent galaxies we find in WINGS are not a complete census in clusters at $z=0.04-0.07$, for several reasons: (1) WINGS typically probe out to about half of the cluster virial radii; (2) WINGS is an X-ray fluxlimited sample, with different flux limits in the Northern and Southern Hemispheres, and it is not complete down to a fixed $\mathrm{X}$-ray luminosity; (3) WINGS does not include clusters with $L_{X}<0.2 \times 10^{44} \mathrm{erg} \mathrm{s}^{-1}$.

This is an indication that the remaining $80 \%$ of quiescent galaxies at high- $z$ and, presumably, some fraction of the SDG population, must be found today in the "field," where the "field" includes the outer regions of WINGS clusters, as well as a large number of low-mass clusters and groups. Hence, we speculate

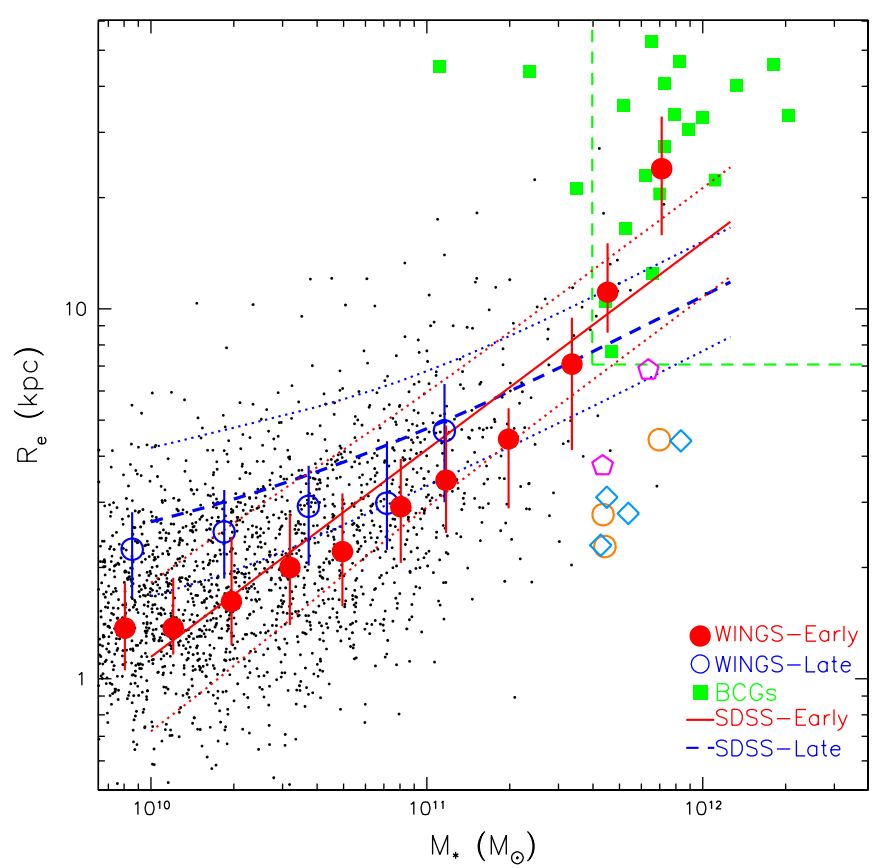

Figure 5. Mass-radius relation for WINGS cluster members (small black dots). Open blue circles are the median values with upper and lower quartiles for late-type (later than S0) galaxies, and red filled circles for early-type (elliptical and S0) galaxies. Green squares are WINGS brightest cluster galaxies (BCGs). The SDSS median and $1 \sigma$ relations from Shen et al. (2003) for early- (red full line, $n>2.5$ ) and late-type (blue dashed line, $n<2.5$ ) galaxies are also drawn. (A color version of this figure is available in the online journal.)

that groups and clusters may host a large fraction or even the totality of high- $z$ quiescent descendants.

\section{WHY LOW- $z$ SDGs GALAXIES WERE NOT FOUND BEFORE}

In Figure 5, we plot the mass-radius relation for $M_{*} \geqslant$ $10^{10} M_{\odot}$ WINGS cluster members (small black dots). The SDSS Shen et al. (2003) relation, commonly used by high- $z$ studies as local reference, is superimposed as a red full line for early-type galaxies (Sersic $n>2.5$ ), and as a blue dashed line for late type $(n<2.5)$, with their $1 \sigma$ limits. Large dots are WINGS median values with upper and lower quartiles (completeness corrected), blue open dots for late-type galaxies (morphologies later than S0), and red filled dots for early-type (elliptical and S0) galaxies. 
Table 2

Local WINGS Mass-Radius Relation

\begin{tabular}{lcc}
\hline \hline $\log _{10}\left(M_{*} / M_{\odot}\right)$ & $\log _{10}\left(R_{e} / \mathrm{kpc}\right)$ & $\log _{10}\left(R_{e}^{\mathrm{SDSS}} / \mathrm{kpc}\right)$ \\
\hline $9.93_{-0.07}^{+0.09}$ & Late-type galaxies & \\
$10.27_{-0.08}^{+0.04}$ & $0.35_{-0.13}^{+0.10}$ & 0.41 \\
$10.57_{-0.10}^{+0.07}$ & $0.40_{-0.13}^{+0.11}$ & 0.48 \\
$10.86_{-0.07}^{+0.06}$ & $0.47_{-0.16}^{+0.11}$ & 0.55 \\
$11.06_{-0.04}^{+0.08}$ & $0.48_{-0.13}^{+0.17}$ & 0.63 \\
& $0.67_{-0.19}^{+0.13}$ & 0.70 \\
\hline $9.91_{-0.06}^{+0.05}$ & Early-type galaxies & \\
$10.08_{-0.03}^{+0.06}$ & $0.14_{-0.11}^{+0.12}$ & 0.01 \\
$10.29_{-0.05}^{+0.06}$ & $0.14_{-0.07}^{+0.14}$ & 0.10 \\
$10.50_{-0.04}^{+0.05}$ & $0.21_{-0.12}^{+0.17}$ & 0.22 \\
$10.69_{-0.05}^{+0.06}$ & $0.30_{-0.16}^{+0.14}$ & 0.34 \\
$10.91_{-0.06}^{+0.04}$ & $0.34_{-0.15}^{+0.16}$ & 0.45 \\
$11.07_{-0.03}^{+0.06}$ & $0.47_{-0.15}^{+0.13}$ & 0.57 \\
$11.30_{-0.07}^{+0.05}$ & $0.54_{-0.14}^{+0.15}$ & 0.66 \\
$11.53_{-0.06}^{+0.03}$ & $0.65_{-0.19}^{+0.08}$ & 0.79 \\
$11.66_{-0.03}^{+0.05}$ & $0.85_{-0.23}^{+0.12}$ & 0.91 \\
$11.85_{-0.01}^{+0.01}$ & $1.05_{-0.11}^{+0.13}$ & 0.99 \\
\hline & $1.38_{-0.18}^{+0.14}$ & 1.10 \\
\hline
\end{tabular}

Notes. The values are logarithm of the median estimates, errors are the lower and upper quartiles of the distributions. The SDSS $R_{e}$ is the expected value calculated from Shen et al. (2003) at the same mass reported in the first column.

Green squares are the BCGs: together with all galaxies with $M_{*}>4 \times 10^{11} M_{\odot}$ these appear to significantly deviate from the general trend of all galaxies, showing a marked steepening of the mass-radius relation at high masses. Indeed they seem to belong to a separate class of objects which likely underwent a significant accretion of cold gas during the formation of the cluster (see, e.g., Bernardi 2009); this was the main reason why we considered inappropriate to include them in the present study. We want to stress here that this is just an empirical upper mass limit that arises from a visual inspection of Figure 5, whose physical explanation is simply tentative and qualitative. With this is mind, we also note that high- $z$ data (symbols and color code are the same of Figure 4$)$ of $M_{*}>4 \times 10^{11} M_{\odot}$ are smaller by a factor of $\geqslant 3$ than the WINGS cluster BCGs. A strong evolution in radius is thus required for BCGs, at odds with the rest of the galaxies.

Importantly, we find systematically lower radii $(\sim 0.1 \mathrm{dex})$ in our cluster early- and late-type galaxies, when compared with SDSS. In Table 2, we report, as reference, our median $R_{e}$ values for different bins in mass, both for early- and late-type galaxies, together with the corresponding SDSS value calculated from Shen et al. (2003). If low- $z$ cluster galaxies are the proper descendants of the high- $z$ ones, $\sim 20 \%$ of the claimed evolution in radius needed to match the local mass-radius relation could be due to the uncorrect choice of the local relation. It is worth noting that the difference in the local mass-radius relation could be almost completely explained with the systematic offset in mass we are finding with SDSS-DR7 masses, at variance with Gallazzi et al. (2005), Hyperzmass, and Bell \& de Jong (2001) (see Section 3.1).

In the following, we briefly discuss the possible causes of systematic errors which one might take into consideration when assessing the problem of how and how much high- $z$ galaxies have undergone structural evolution.
Galaxy stellar masses. As discussed in Section 2, when comparing different data sets it is of paramount importance to ensure consistency on the IMF assumed and, at high- $z$, on the model prescriptions. IMF slopes and limits have to be carefully matched. At high-z, it is also important to homogenize the treatment of the stellar TP-AGB phase, since the masses of stellar populations with ages of approximately $2 \mathrm{Gyr}$, can be overestimated by $\sim 0.15$ dex (Maraston 2005; Cimatti et al. 2008). Furthermore, the type of stellar mass considered is crucial (see Section 2). At high-z, the mass locked into stellar remnants is negligible, while it becomes more and more important at lower$z$ (up to $\sim 0.15$ dex). Hence, the comparison with low $-z$ masses, to be meaningful, should be carried out considering the mass locked in remnants at low- $z$ (item (2) in Section 2.2.1).

Effective-radius. We note that Blanton et al. (2005) discuss a bug in the 2003 tool which measured the sizes for the NYU-VAGC catalog that caused small radii to be overestimated; these sizes were used by Shen et al. (2003) for the SDSS mass-radius relation. While we are not able to estimate the importance of this effect, the corrected radii should clearly be used to reassess the SDSS mass-radius relation. Furthermore, a recent paper by Guo et al. (2009) studies the possible biases induced by a noisy background subtraction.

In a recent paper, Mancini et al. (2009) claim that $R_{e}$ measurements of low $\mathrm{S} / \mathrm{N}$ high- $z$ compact galaxies, may give systematic lower sizes up to a factor of $\sim 2$.

Extreme selection criteria. The definition of a "SDG" is necessarily arbitrary at some level. Trujillo et al. (2009) search for SDGs with $M_{*} \geqslant 8 \times 10^{10} M_{\odot}$ and $R_{e} \leqslant 1.5 \mathrm{kpc}$, and found no candidates with old luminosity-weighted ages, and very few of all ages. Only $9 \%$ of the high- $z$ galaxies (10 out of 108) considered in our analysis fulfill these extreme mass and radius selection criteria. Interestingly, we found approximately the same fraction $(\sim 5 \%)$, applying this definition in our sample: we have only 16 "extreme" SDGs, with a median luminosity-weighted age of 10.1 Gyr. Hence, it is plausible that only a small fraction of galaxies satisfies such extreme criteria.

Completeness. In a recent paper, Taylor et al. (2009) perform a thoroughly search of SDGs in the $z \sim 0.1$ SDSS galaxies. In particular, the authors discuss the different varieties of incompletenesses involved when using SDSS data to assess a proper local mass-radius relation. Fiber collision and/or limit in surface brightness when selecting galaxies for spectroscopic follow-up, could cause SDSS to miss local clusters galaxies in a systematic way. However, the authors show that completeness issues are not sufficient to explain the lack of galaxies as compact as those in the van Dokkum et al. (2008) sample, which do not have a local counterpart in WINGS too (see Section 4). We refer to that paper for the details on their analysis of massive compact galaxies in the SDSS.

\section{CLUSTER SDGs PROPERTIES: CLUES TO THEIR ORIGIN}

The WINGS SDGs sample consists of 31 ellipticals, 78 S0s, and 13 late-type galaxies. When completeness corrected, these numbers become $46.5(22.8 \% \pm 4 \%), 136.9(67.3 \% \pm 7 \%)$, and $20.1(9.9 \% \pm 2 \%)$, respectively. If compared with the overall morphological fractions in a magnitude limited sample (see Poggianti et al. 2009, Figure 1 and Table 1), there is an excess of S0s at the expense of ellipticals and later types (see Table 3). This might indicate either that the S0 morphology is preferred by SDGs, or that some of these S0s have uncorrect $R_{e}$ because their light profile is not well suited for a single Sersic law fit. 
Table 3

Characteristic Numbers of WINGS SDGs

\begin{tabular}{lcc}
\hline \multicolumn{1}{c}{ Quantity } & Value & rms error \\
\hline SDGs & 122 & 11 \\
SDGs C.C. & 203.5 & 14.3 \\
$\left\langle R_{e}\right\rangle$ & 1.61 & 0.29 \\
$\langle n\rangle$ & 3.0 & 0.6 \\
$\langle b / a\rangle$ & 0.54 & 0.18 \\
$\left\langle M_{*}\right\rangle$ & $8.7 \times 10^{10} M_{\odot}$ & $2.5 \times 10^{10} M_{\odot}$ \\
$\left\langle V_{\text {abs }}\right\rangle$ & -20.68 & 0.37 \\
$\langle$ Lw-age $\rangle$ & 9.62 & 1.94 \\
$\langle$ Mw-age $\rangle$ & 12.02 & 1.28 \\
Ellipticals fraction C.C. & $22.8 \%$ & $\ldots$ \\
S0s fraction C.C. & $67.3 \%$ & $\ldots$ \\
Late-type fraction C.C. & $9.9 \%$ & $\ldots$ \\
\hline
\end{tabular}

Note. Errors are derived from Poissonian statistics for counts, and are rms for other quantities; C.C.: completeness corrected.

In Figure 6, we present the distributions of several relevant quantities describing our SDGs sample. First of all, the axial ratio distribution $(\langle b / a\rangle=0.54 \pm 0.18)$ shows that WINGS SDGs have a tendency to be flattened, mostly due to the high fraction of S0s. As expected, the population of elliptical galaxies is remarkably rounded. The late-type galaxies are extremely flat and could, in principle, introduce systematics in our analysis. We decided to keep them because most of the high- $z$ samples are not selected on the basis of their morphology and therefore may include late-type galaxies. Anyway, all the conclusions of our present study are even reinforced if only early-type galaxies are considered.

The Sersic indexes of our SDGs are characteristic of disky like rather than of early-type galaxies $(\langle n\rangle=3.0 \pm 0.6)$ : while late-type SDGs present an expected $n \sim 1$ value, the majority of elliptical SDGs have remarkably low values too.

The WINGS median $R_{e}$ is similar to that of high- $z$ SDGs $\left(\left\langle R_{e}\right\rangle=1.61 \pm 0.29\right)$, with a few of them being larger than $2.5 \mathrm{kpc}$. These objects are the most massive ones, and they probably are transition objects from the compact phase to a more complex radius inflation phase, where most probably galaxies acquire gas and/or stars in the external regions, increasing $R_{e}$ (see Figure 5).

Our SDGs have high intrinsic luminosity $\left\langle M_{V}\right\rangle=-20.68 \pm$ 0.37 , and stellar masses $\left\langle M_{*}\right\rangle=(8.67 \pm 2.55) \times 10^{10} M_{\odot}$.

WINGS SDGs may show a slight tendency to prefer the central regions of clusters (CCD) and intermediate/high-density regions (LD), but overall their clustercentric and local density distribution are not too dissimilar from those of galaxies of similar mass. Our images are uniformly sampling the cluster populations as far as $R_{500}\left(\sim 0.6 R_{200}\right)$ for all clusters, so the sharp decline at larger radii may be just a result of the area coverage. A future ancillary project with the forthcoming OMEGACAM at the VLT Survey Telescope will survey a considerable fraction of WINGS clusters at much larger radii, and will uncover possible compact candidates at larger distances.

We have already discussed the ages of our SDGs in Section 3, showing the difference between Lw-age and Mw-age, and the care that needs to be taken when using these quantities to select
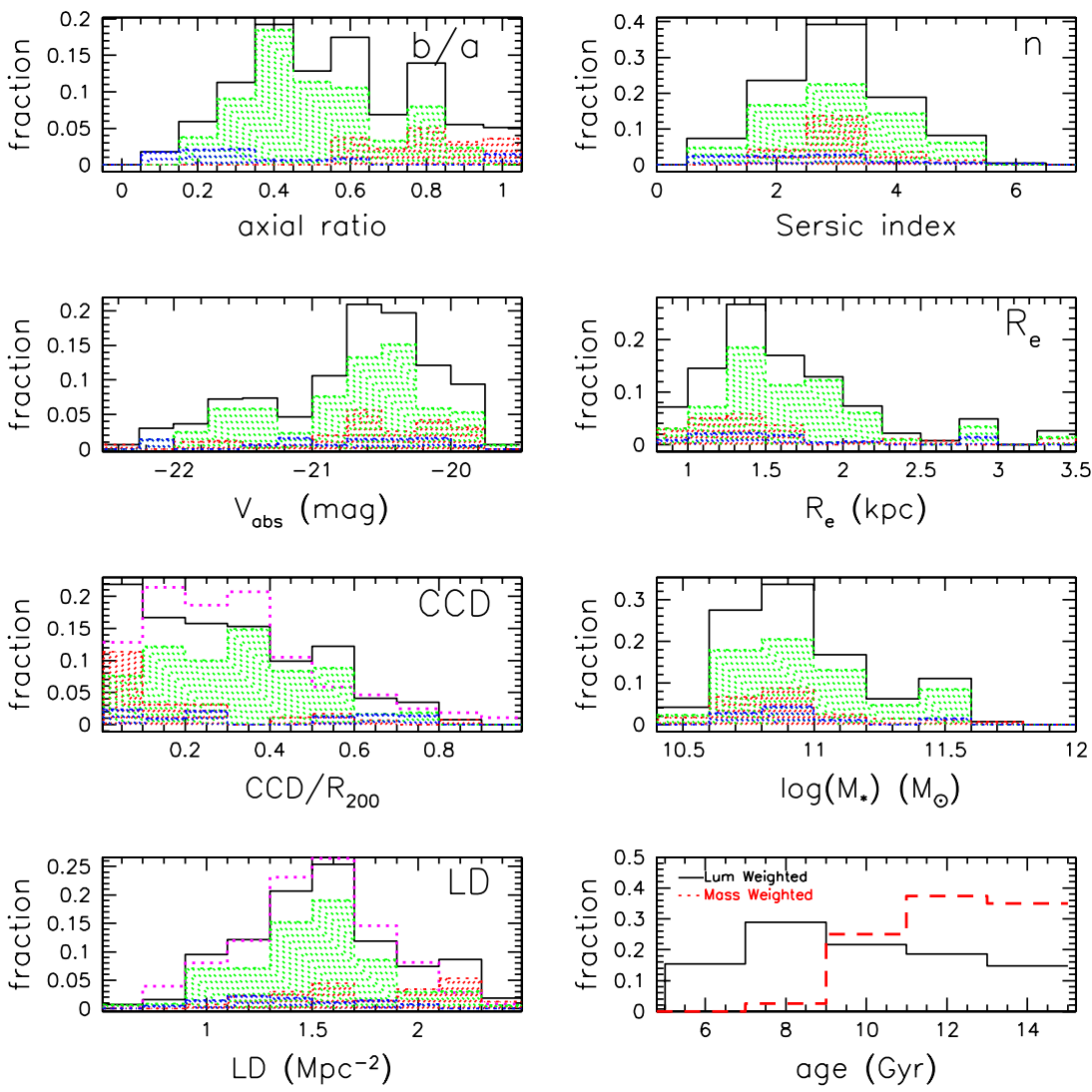

Figure 6. Distributions of different quantities of interest for WINGS SDGs cluster members. The color shades correspond to morphologies—red: ellipticals, green: SOs, and blue: late-type galaxies. $M_{*}$ is the total stellar mass (Fritz et al. 2007), CCD is the cluster centric distance in units of $R_{200}$ (Carlberg et al. 1997), while LD is the local density (Dressler 1980). The magenta dashed histograms are the distribution of non-compact galaxies in the same mass range of SDGs.

(A color version of this figure is available in the online journal.) 


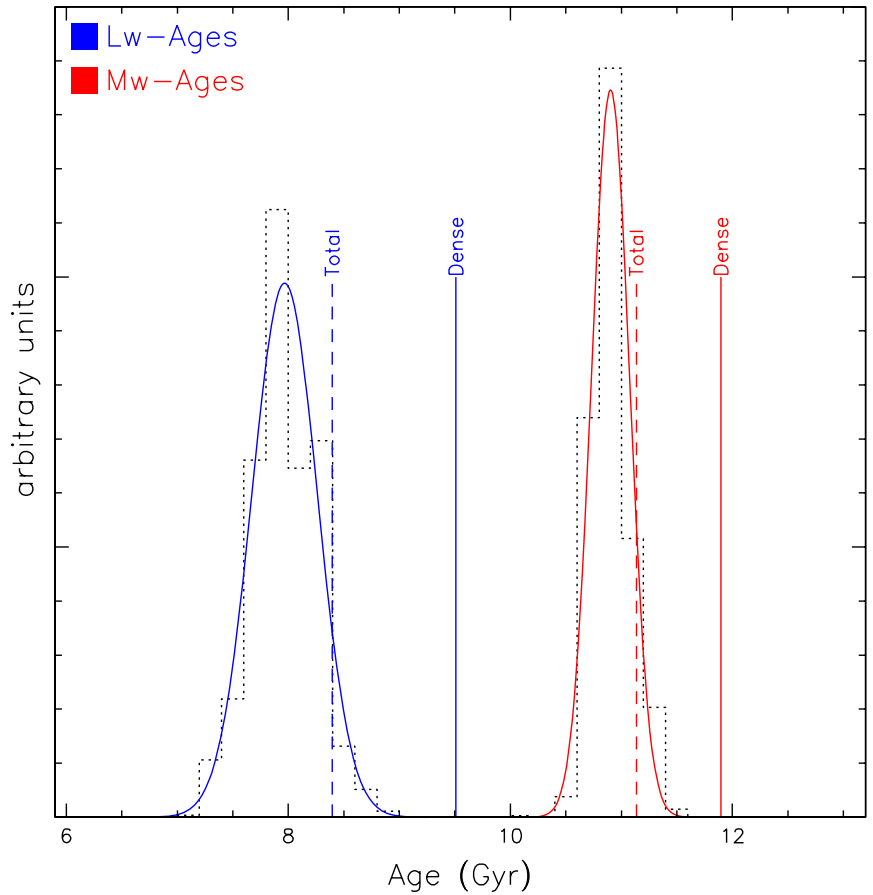

Figure 7. Distributions of median luminosity-weighted ages (left blue histogram) and mass-weighted ages (right red histogram) of 1000 random extractions of non-superdense early-type galaxies with the same mass distribution of SDGs. The vertical lines are the median ages of SDGs (solid lines) and of non-superdense galaxies (dashed lines) with their intrinsic mass distribution.

(A color version of this figure is available in the online journal.)

samples. WINGS SDGs have high median Lw- $(\langle$ Lw-age $\rangle=$ $9.6 \pm 1.9 \mathrm{Gyr})$ and Mw-ages $(\langle\mathrm{Mw}-\mathrm{age}\rangle=12.0 \pm 1.3 \mathrm{Gyr})$, showing that the bulk of the mass has an age typically $2 \mathrm{Gyr}$ older than the luminosity-weighted age (red dashed histogram in Figure 6).

To quantify to what extent, on average, WINGS SDGs are older than non-compact galaxies of similar masses, we used the Monte Carlo technique to extract 1000 random samples of "normal" galaxies with the same mass distribution of the SDGs. This is done to disentangle the dependence of age from mass. We used the early-type galaxies only, to be more conservative, as we know that there are more late types among "normal" galaxies than in the SDGs sample. In Figure 7, we plot the distributions of the median Lw- (blue lines) and Mw-ages (red lines) of these 1000 samples. Choosing "normal galaxies" according to the SDG mass distribution gives on average younger ages than those of all (i.e., not mass matched) "normal" galaxies (vertical dashed lines). Importantly, the Monte Carlo simulation shows that compact galaxies tend to be $\sim 1.5$ Gyr older (both in Lw and Mw) than normal galaxies of the same mass, again suggesting that in some way age is related to compactness, in addition than mass, as we discussed regarding Figure 2.

We have seen that the WINGS SDGs sample consists of galaxies similar in all respects to the compact quiescent ones found in recent high- $z$ studies. They are very old, massive, and compact. Their presence in the local universe opens new perspectives on their formation and evolution, which may change the present understanding of their nature.

\section{SUMMARY AND CONCLUSIONS}

We find 122 SDGs in the WINGS survey of nearby galaxy clusters $(z \sim 0.05)$, with stellar mass $3 \times 10^{10} \leqslant M_{*} / M_{\odot} \leqslant$
$4 \times 10^{11}$ and surface mass density $\Sigma_{50} \geqslant 3 \times 10^{9} M_{\odot} \mathrm{kpc}^{-2}$. They represent nearly $22 \%$ of all cluster members in the same mass range. They have masses and sizes similar to their high- $z$ counterparts.

We find that both mass and radius determine the age of massive low $z$ cluster galaxies: the larger the mass, and the smaller the radius, the older the stellar population. Selecting quiescent galaxies at any redshift results in selecting the smallest galaxies; the further back in time we search for quiescent galaxies, the smaller the sizes we measure as a consequence of this effect.

We compare our data with spectroscopic high- $z$ studies, whose mass and size estimates are more reliable than photometric ones. If cluster galaxy sizes and masses today are representative of those of high- $z$ galaxies, our findings show that there is no need for an evolution in size (at least as far as $z \sim 2$ ), when this age effect is properly taken into account. The largest possible evolution in size at $z<2$, based on dynamical and therefore possible dishomogeneous mass measurements, is very mild, a factor of 1.5 at most, much smaller of the factor 3 claimed in the literature; anyway, it is difficult to directly interpret this discrepancy due to the different way masses are estimated. In contrast, there is strong evidence for a large evolution in radius for the most massive galaxies with $M_{*}>4 \times 10^{11} M_{\odot}$ compared to similarly massive galaxies, in WINGS, i.e., the BCGs.

On the other hand, the sizes of galaxies in the sample of van Dokkum et al. (2008) at $z=2.4$ are smaller by a factor of $\sim 3$ even with respect to the WINGS data points in Figure 4, when the age-selection effect is taken into account. These objects represent a population of galaxies visibly different from other high- $z$ data (see Figure 1).

For masses $M_{*} \leqslant 3 \times 10^{10} M_{\odot}$, there is a significant decrease in the frequency of SDGs and speculate that this rapid decrease in number could be an indication that a minimum threshold in mass is required to form compact galaxies.

The local mass-radius relation by Shen et al. (2003), used by high- $z$ studies as a reference, turns out to be shifted toward higher radii at fixed mass when compared to the WINGS relation. This is probably due to the systematic offset between our total masses with respect to SDSS-DR7 masses, discussed in Section 3.1; anyway, our masses turn out to be in good agreement with SDSS-DR4 (Gallazzi et al. 2005), Hyperzmass, and Bell $\&$ de Jong (2001).

Assuming that SDGs reside only in clusters, we calculate a hard lower limit of their number density in the nearby universe of $1.3 \times 10^{-5} \mathrm{Mpc}^{-3}$, which becomes $0.57 \times 10^{-5} \mathrm{Mpc}^{-3}$ if only SDGs that were quiescent at $z \sim 1.5$ (i.e., luminosity-weighted age $\geqslant 10 \mathrm{Gyr}$ ) are considered. While no published data on high$z$ SDGs number density are available, there are estimates for the density of quiescent galaxies at $z \sim 1.5$. We find a lower limit of $0.18 \times 10^{-4} \mathrm{Mpc}^{-3}$ of such quiescent galaxies with $M_{*}>4 \times 10^{10} M_{\odot}$ in clusters, to be compared with a high$z$ value of $10^{-4} \mathrm{Mpc}^{-3}$ (Wuyts et al. 2009). Around $20 \%$ of all high- $z$ quiescent galaxies are therefore found in the inner regions of WINGS clusters.

Our findings challenge the simple picture of a widespread evolution of the radius of compact high- $z$ galaxies with redshift. The presence of compact galaxies in local clusters suggests that the formation and evolution of such systems may not be simply explained with a "growing-radius" mechanism, as presently thought. In particular, our research can be used to further constrain the current picture of galaxy mass assembly (hierarchical merging, down-sizing, etc.) from the first few 
gigayears after the big bang, to the present galaxy clusters and high-density regions probed by the WINGS survey.

We thank Micol Bolzonella, Alessandro Bressan, Michele Cappellari, Anna Gallazzi, Laura Greggio, Laura Portinari, Alvio Renzini, Paolo Saracco, Edward Taylor, and Ignacio Trujillo for useful input and discussions. We acknowledge financial support from the Astronomy Department of the University of Padova and INAF-National Institute for Astrophysics through its PRIN-INAF2006 scheme.

Facilities: ING:Newton (WFC), AAT(2dF), ING:Herschel (WYFFOS)

\section{REFERENCES}

Bell, E. F., \& de Jong, R. S. 2001, ApJ, 550, 212

Bernardi, M. 2009, MNRAS, 395, 1491

Bezanson, R., van Dokkum, P. G., Tal, T., Marchesini, D., Kriek, M., Franx, M., \& Coppi, P. 2009, ApJ, 697, 1290

Blanton, M. R., et al. 2005, AJ, 129, 2562

Bolzonella, M., Miralles, J.-M., \& Pelló, R. 2000, A\&A, 363, 476

Buitrago, F., Trujillo, I., Conselice, C. J., Bouwens, R. J., Dickinson, M., \& Yan, H. 2008, ApJ, 687, L61

Cappellari, M., et al. 2006, MNRAS, 366, 1126

Cappellari, M., et al. 2009, ApJ, 704, L34

Cardelli, J. A., Clayton, G. C., \& Mathis, J. S. 1989, ApJ, 345, 245

Carlberg, R. G., Morris, S. L., Yee, H. K. C., \& Ellingson, E. 1997, ApJ, 479, L19

Cava, A., et al. 2009, A\&A, 495, 707

Cenarro, A. J., \& Trujillo, I. 2009, ApJ, 696, L43

Cid Fernandes, R., Mateus, A., Sodré, L., Stasińska, G., \& Gomes, J. M 2005, MNRAS, 358, 363

Cimatti, A., et al. 2008, A\&A, 482, 21

Conselice, C. J. 2003, ApJS, 147,

Conselice, C. J., Bershady, M. A., \& Jangren, A. 2000, ApJ, 529, 886

Daddi, E., et al. 2005, ApJ, 626, 680

Damjanov, I., et al. 2009, ApJ, 695, 101

de Vaucouleurs, G. 1974, in IAU Symp. 58, The Formation and Dynamics of Galaxies, ed. J. R. Shakeshaft (Dordrecht: Kluwer), 1

Dressler, A. 1980, ApJ, 236, 351

Ebeling, H., Edge, A. C., Allen, S. W., Crawford, C. S., Fabian, A. C., \& Huchra, J. P. 2000, MNRAS, 318, 333

Ebeling, H., Voges, W., Bohringer, H., Edge, A. C., Huchra, J. P., \& Briel, U. G. 1996, MNRAS, 281, 799

Ebeling, H., et al. 1998, MNRAS, 301, 881

Fan, L., Lapi, A., De Zotti, G., \& Danese, L. 2008, ApJ, 689, L101

Fasano, G., et al. 2006, A\&A, 445, 805
Fritz, J., et al. 2007, A\&A, 470, 137

Gallazzi, A., Charlot, S., Brinchmann, J., White, S. D. M., \& Tremonti, C. A. 2005, MNRAS, 362, 41

Graves, G. J., Faber, S. M., \& Schiavon, R. P. 2009, ApJ, 693, 486

Guo, Y., et al. 2009, MNRAS, 398, 1129

Hopkins, P. F., Bundy, K., Murray, N., Quataert, E., Lauer, T. R., \& Ma, C. 2009a, MNRAS, 398, 898

Hopkins, P. F., Hernquist, L., Cox, T. J., Keres, D., \& Wuyts, S. 2009b, ApJ, 691,1424

Jacoby, G. H., Hunter, D. A., \& Christian, C. A. 1984, ApJS, 56, 257

Joung, M. R., Cen, R., \& Bryan, G. L. 2009, ApJ, 692, L1

Kauffmann, G., et al. 2003, MNRAS, 341, 33

Khochfar, S., \& Silk, J. 2006, ApJ, 648, L21

Kriek, M., van Dokkum, P. G., Franx, M., Illingworth, G. D., \& Magee, D. K 2009, ApJ, 705, L71

Kroupa, P. 2001, MNRAS, 322, 231

Longhetti, M., \& Saracco, P. 2009, MNRAS, 394, 774

Mancini, C., Matute, I., Cimatti, A., Daddi, E., Dickinson, M., Rodighiero, G., Bolzonella, M., \& Pozzetti, L. 2009, A\&A, 500, 705

Maraston, C. 2005, MNRAS, 362, 799

Marleau, F. R., \& Simard, L. 1998, ApJ, 507, 585

Peng, C. Y., Ho, L. C., Impey, C. D., \& Rix, H.-W. 2002, AJ, 124, 266

Pignatelli, E., Fasano, G., \& Cassata, P. 2006, A\&A, 446, 373

Poggianti, B. M., et al. 2009, ApJ, 697, L137

Salpeter, E. E. 1955, ApJ, 121, 161

Sanchez-Blazquez, P. 2004, PhD thesis, Universidad Complutense de Madrid

Sánchez-Blázquez, P., et al. 2006, MNRAS, 371, 703

Saracco, P., Longhetti, M., \& Andreon, S. 2009, MNRAS, 392, 718

Shankar, F., \& Bernardi, M. 2009, MNRAS, 396, L76

Shen, S., Mo, H. J., White, S. D. M., Blanton, M. R., Kauffmann, G., Voges, W., Brinkmann, J., \& Csabai, I. 2003, MNRAS, 343, 978

Taylor, E. N., Franx, M., Glazebrook, K., Brinchmann, J., van der Wel, A., \& van Dokkum, P. G. 2009, ApJ, submitted (arXiv:0907.4766)

Toft, S., et al. 2007, ApJ, 671, 285

Trujillo, I., Cenarro, A. J., de Lorenzo-Cáceres, A., Vazdekis, A., de la Rosa I. G., \& Cava, A. 2009, ApJ, 692, L118

Trujillo, I., Conselice, C. J., Bundy, K., Cooper, M. C., Eisenhardt, P., \& Ellis, R. S. 2007, MNRAS, 382, 109

Trujillo, I., et al. 2006, ApJ, 650, 18

Valentinuzzi, T., et al. 2009, A\&A, 501, 851

van der Wel, A., Bell, E. F., van den Bosch, F. C., Gallazzi, A., \& Rix, H.-W. 2009, ApJ, 698, 1232

van der Wel, A., Holden, B. P., Zirm, A. W., Franx, M., Rettura, A., Illingworth, G. D., \& Ford, H. C. 2008, ApJ, 688, 48

van Dokkum, P. G., Kriek, M., \& Franx, M. 2009, Nature, 460, 717

van Dokkum, P. G., et al. 2008, ApJ, 677, L5

Varela, J., et al. 2009, A\&A, 497, 667

Wuyts, S., et al. 2009, ApJ, 700, 799

Zirm, A. W., et al. 2007, ApJ, 656, 66 